\title{
Campos Elétrico e Magnético em Linhas de Transmissão de Energia
}

\section{Resumo}

Este artigo apresenta uma nova abordagem para o cálculo de campos elétricos e magnéticos de linhas aéreas de transmissão, ao utilizar "O método de simulação da carga ..." [1]. Neste caso, a análise do potencial elétrico no tempo, o cálculo das cargas instantâneas, e a utilização de correntes instantâneas, permitem obter os valores instantâneos dos campos. Os resultados obtidos pelo programa de computador para calcular os perfis do campo elétrico e do campo magnético ao nível do solo da linha aérea de transmissão Batalha - Pego $400 \mathrm{kV}$ são comparados com as medições efetuadas nessa linha [2]. Podemos concluir que a simulação no tempo é mais precisa e dá valores consistentes com a realidade. O programa de cálculo desenvolvido pode ser utilizado com todas as possíveis configurações das linhas de transmissão. A aplicação do programa noutras linhas aéreas de transmissão permite a obtenção de conclusões interessantes sobre a questão da segurança e da saúde das pessoas. As simulações sugerem bons resultados sendo confirmados através das medições efetuadas noutras linhas de energia [2] [3].

\section{Palavras-chave}

Linha Aérea de Transmissão de Energia, Campo Elétrico, Campo Magnético, Método de Simulação da Carga, Valores Instantâneos, Limites de Exposição, Zona de Segurança.

\section{Introdução}

As Linhas Aéreas de Transmissão (LAT) criam campos elétricos e magnéticos cujos valores podem por em causa a segurança e a saúde do público geral ou população sujeita a exposições frequentes ou permanentes.
A determinação dos valores previsíveis dos campos a alturas próximas do solo $(\approx 1 \mathrm{~m})$ na faixa de segurança e até alguns metros para lá é fundamental para definir restrições e níveis de exposição da população a campos eletromagnéticos.

A metodologia utilizada neste trabalho para cálculo do campo elétrico em LAT baseia-se no Método de Simulação de Cargas [1]. Com a particularidade de os campos serem calculados no tempo, devido à atual capacidade de cálculo dos programas computacionais, obtêm-se valores teóricos mais consentâneos com a realidade.

As medições utilizadas [2] e [4] para comparação com os valores calculados fazem parte do estudo da LAT Batalha Pego, 400 kV (perfil 10) e da LAT RM/PR - Batalha2 (perfil 3).

\section{Campo Elétrico}

O Método de Simulação de Cargas [1] consiste na substituição da distribuição real de cargas elétricas na superfície do condutor, a um determinado potencial, por $n$ linhas de cargas fictícias colocadas no interior do condutor.

A simplicidade geométrica dos condutores de uma LAT permite considerar uma única linha de carga, cuja dimensão equivalente é dada pelo raio médio geométrico do condutor ou da fase se constituída por vários condutores, o que simplifica os cálculos.

O cálculo dos campos elétricos efetua-se a partir do conhecimento das cargas elétricas em cada uma das fases da linha e nos cabos de guarda.

O solo é assumido como um condutor perfeito, o tempo para que as cargas se redistribuam na sua superfície é extremamente pequeno $(0,1$ a $100 \eta \mathrm{s})$ e é menor que o tempo de relaxação $(\tau=\rho \varepsilon)$, comparado com a frequência do sistema de energia. 
Os valores das cargas instantâneas, matriz [q], são obtidos a partir do potencial instantâneo nos condutores, matriz [v], e pelo coeficiente do potencial de Maxwell, matriz [P], na solução da equação:

$[q]=[P]^{-1}[v]$

É utilizado o modelo bidimensional para o cálculo do perfil de variação do campo, no plano vertical perpendicular à direção da LAT. Este modelo, relativamente simples, é de rigor adequado.

Para LAT cujos condutores são paralelos $(i, j, \ldots)$ sobre um solo perfeitamente plano, os elementos da matriz [P] são dados pelas equações;

$P_{i i}=\frac{1}{2 \pi \varepsilon_{0}} \ln \left(\frac{2 y_{i}}{r_{i}^{*}}\right)$

$P_{i j}=\frac{1}{2 \pi \varepsilon_{0}} \ln \left[\frac{\left(x_{i}-x_{j}\right)^{2}+\left(y_{i}+y_{j}\right)^{2}}{\left(x_{i}-x_{j}\right)^{2}+\left(y_{i}-y_{j}\right)^{2}}\right]^{\frac{1}{2}}$

Onde; yi é a distância do condutor ao solo, xi e a posição horizontal do condutor em relação ao eixo da LAT e $r_{i}^{*}$ o raio médio geométrico do condutor, para os condutores $(i, j, \ldots)$.

Para as fases com feixe de condutores o raio médio geométrico é dado por [5],

$r_{i}^{*}=\sqrt[m]{m r_{m g} R^{m-1}}$

$R=\frac{d}{2 \operatorname{sen}(\pi / m)}$

Onde; $m$ é o número de condutores do feixe, $r_{m g}$ é o raio médio geométrico de cada condutor e $d$ a distância entre condutores adjacentes.
Resolvida a equação (1) e determinada a carga nas fases e cabos de guarda, pode-se calcular o campo elétrico instantâneo devido a cada condutor no ponto $\mathrm{N}$ de coordenadas $(x \mathrm{~N}, y \mathrm{~N})$ no espaço, onde o campo elétrico devido a carga no condutor $\mathrm{i}$, e à sua imagem no solo é:

$\bar{E}_{i}=E_{x, i} \bar{u}_{x}+E_{y, i} \bar{u}_{y}$

$\bar{u}_{x}$ e $\bar{u}_{y}$ são os vetores unitários horizontal e vertical e as componentes respectivas de (7) (8) são dados por;

$$
\begin{aligned}
E_{x, i} & =\frac{q_{i}\left(x_{N}-x_{i}\right)}{2 \pi \varepsilon_{0}\left[\left(x_{N}-x_{i}\right)^{2}+\left(y_{N}-y_{i}\right)^{2}\right]} \\
& -\frac{q_{i}\left(x_{N}-x_{i}\right)}{2 \pi \varepsilon_{0}\left[\left(x_{N}-x_{i}\right)^{2}+\left(y_{N}+y_{i}\right)^{2}\right]} \\
E_{y, i} & =\frac{q_{i}\left(y_{N}-y_{i}\right)}{2 \pi \varepsilon_{0}\left[\left(x_{N}-x_{i}\right)^{2}+\left(y_{N}-y_{i}\right)^{2}\right]} \\
& -\frac{q_{i}\left(y_{N}+y_{i}\right)}{2 \pi \varepsilon_{0}\left[\left(x_{N}-x_{i}\right)^{2}+\left(y_{N}+y_{i}\right)^{2}\right]}
\end{aligned}
$$

As componentes horizontal (9) e vertical (10) do campo elétrico, são calculadas pelo somatório das contribuições de todos os condutores $(i, j, \ldots)$.

Sendo que cada uma dessas componentes é um vetor alternado sinusoidal,

$$
E_{x, N}=\sum_{i} E_{x, i}
$$

$E_{y, N}=\sum_{i} E_{y, i}$

o módulo instantâneo do campo é definido por:

$$
E_{N}=\sqrt{E_{x, N}^{2}+E_{y, N}^{2}}
$$


O campo é girante, descrevendo uma trajetória pulsante elíptica no plano transversal (xy).

É possível calcular os valores máximos instantâneos, tanto do vetor campo, como das suas componentes no plano, assim como os respetivos valores eficazes.

\section{Campo Magnético}

O cálculo do perfil do campo magnético também é efetuado para o plano vertical e perpendicular à linha, as correntes instantâneas têm a direção do eixo z. O campo magnético instantâneo no ponto $\mathrm{N}$ é dado pela sobreposição vetorial dos campos criados pelas correntes nos condutores de fase (12).

$\bar{B}_{N}=\frac{\mu_{0}}{2 \pi} \sum_{i} \frac{\overline{i_{i}} \times \bar{r}_{i N}}{r_{i N}^{2}}=\frac{\mu_{0}}{2 \pi} \sum_{i} \frac{i_{i}}{r_{i N}} \bar{\phi}_{i N}$

$\bar{r}_{i N}$ é o vetor de posição do ponto $\mathrm{N}$ em relação à corrente i, cujo módulo é

$r_{i N}=\sqrt{\left(x_{N}-x_{i}\right)^{2}+\left(y_{N}-y_{i}\right)^{2}}$ e $\bar{\phi}_{i N}$ é o vetor unitário do produto externo do vetor corrente com o vetor de posição

$\bar{\phi}_{i N}=-\frac{y_{N}-y_{i}}{r_{i N}} \bar{u}_{x}+\frac{x_{N}-x_{i}}{r_{i N}} \bar{u}_{y}$

As correntes de retorno pela terra são desprezadas, tal com as correntes nos cabos de guarda que são nulas em situação de equilíbrio estacionário, como o solo é considerado um condutor perfeito a corrente de retorno de sistemas elétricos trifásicos simétricos é nula.

\section{Linha Aérea de Transmissão Batalha-Pêgo}

Para a LAT em estudo, $400 \mathrm{kV}(50 \mathrm{~Hz})$ de configuração horizontal, foram calculados os perfis do campo elétrico e do campo magnético, no plano vertical transversal à linha, tomando como parâmetros as características de projeto no local em que foram executadas as medições, e obtidas as alturas cabo - solo do perfil 10 do RL 0718/07 - AT [2].

O gráfico da Fig.1 mostra os valores máximos calculados para as componentes do campo elétrico, à altura de 1,8 metros.

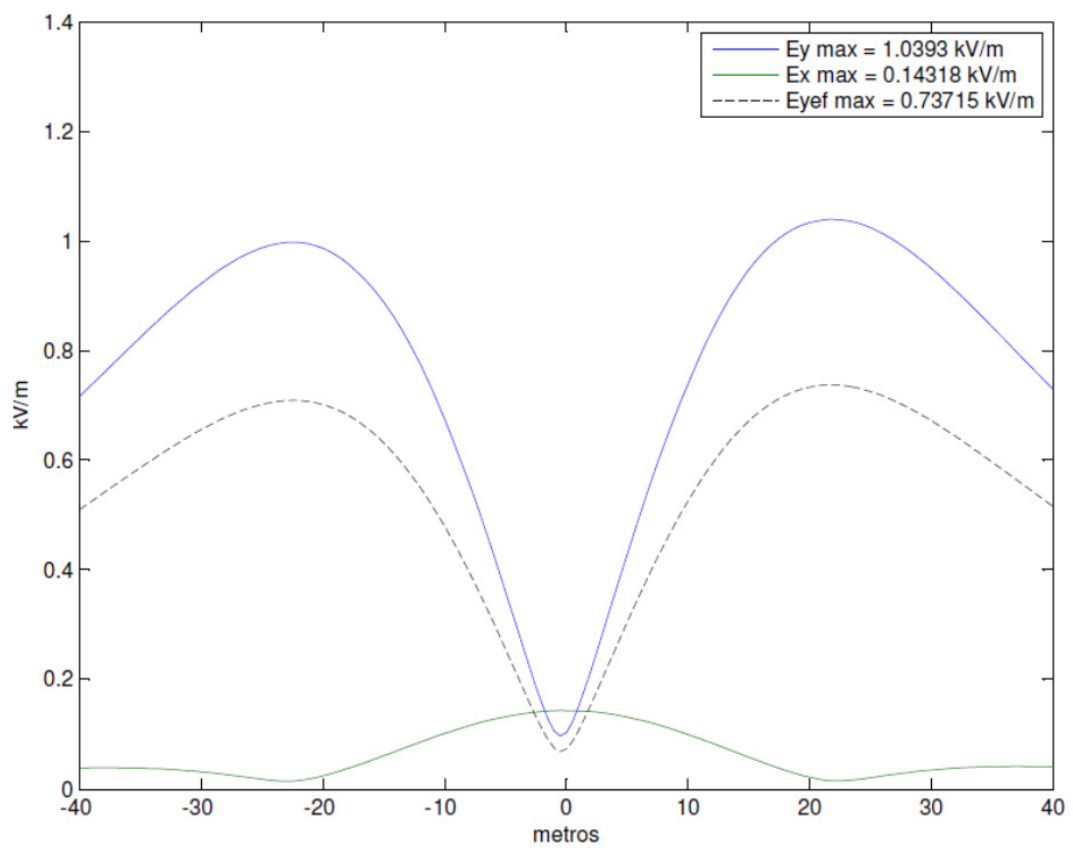

Figura 1 - Perfil do campo elétrico para valores calculados - componentes vertical e horizontal 
O valor máximo do campo verifica-se no ponto $22 \mathrm{~m}$, $E_{\max }=1,04 \mathrm{kV} / \mathrm{m}$ sendo o valor eficaz máximo $E_{\text {ef max }}=0,74$ $\mathrm{kV} / \mathrm{m}$.

Para as medições dos campos foram utilizados instrumentos apropriados para medições de LAT e de acordo com as Normas CEI de ensaio e medição [2].

As medições foram executadas no eixo perpendicular ao sentido longitudinal da LAT, no vão entre as torres 66 e 67 (perfil 10) e em pontos espaçados de $2 \mathrm{~m}$.
O equipamento de medida indica os valores máximos dos campos, em valor eficaz (RMS) [2].

O gráfico da Fig.2 mostra os valores medidos e os valores calculados para o campo elétrico.

As condições de medição dos campos não foram as excelentes.

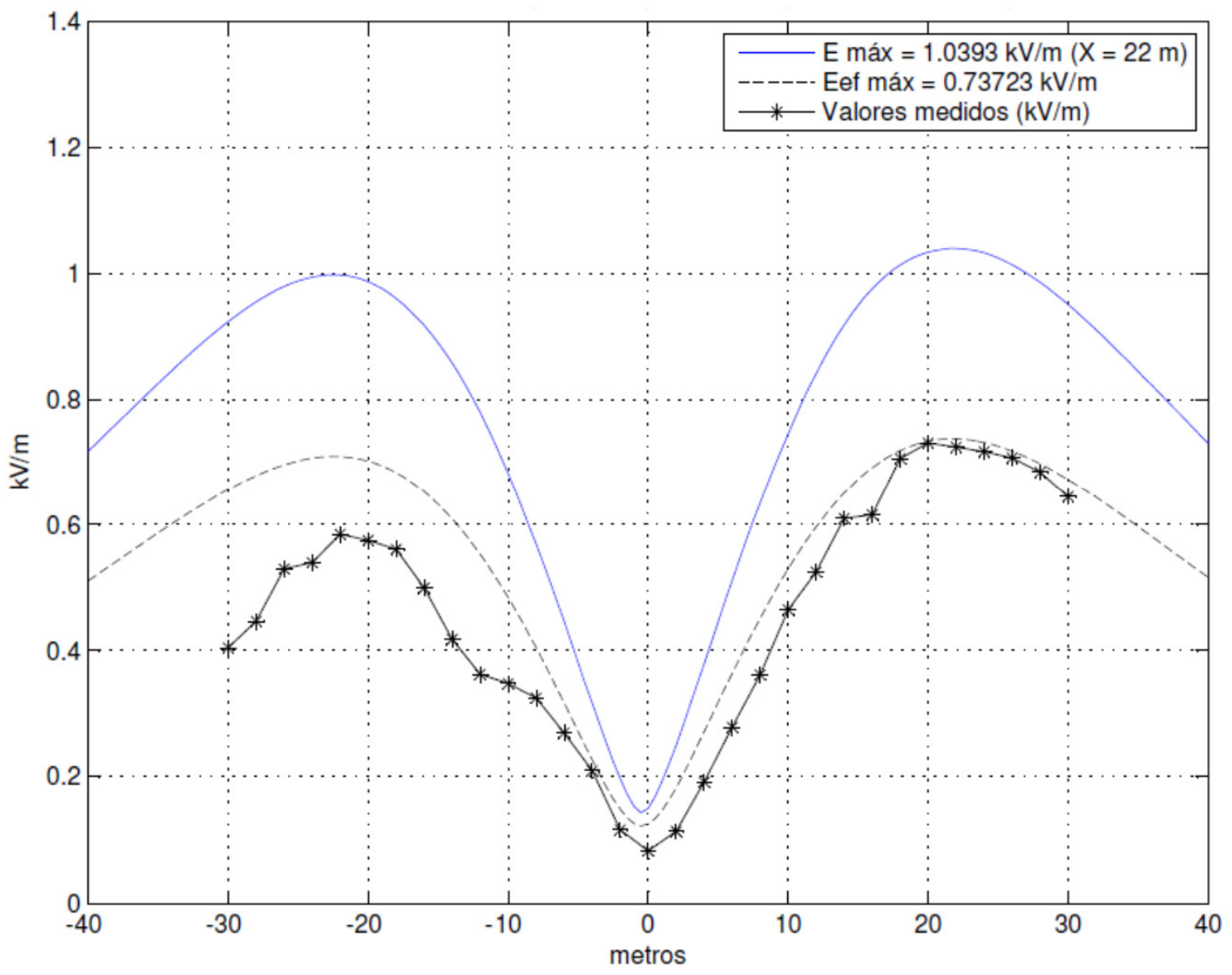

Figura 2 - Perfil do campo elétrico para os valores medidos e calculados 


\section{ARTIGO TÉCNICO}

$\mathrm{Na}$ análise dos valores calculados e medidos do perfil do campo elétrico verifica-se que as maiores discrepâncias encontradas (Fig.3) são devido à existência de um declive de cerca de $2 \mathrm{~m}$ entre a LAT e o ponto -40 e a um muro que acompanha o perfil e funciona como ecrã do campo [2]. Na generalidade as discrepâncias podem ser explicadas por se ter considerado um solo de condução perfeita, plano e horizontal e de extensão infinita.
Se for considerado que as discrepâncias são devidas às condições em que foram efetuadas as medições, os valores podem ser considerados excelentes.

Na Fig.4 apresenta-se a trajetória descrita pelo vetor campo elétrico, no ponto $\mathrm{xN}=0 \mathrm{~m}(\mathrm{yN}=1,8 \mathrm{~m})$.

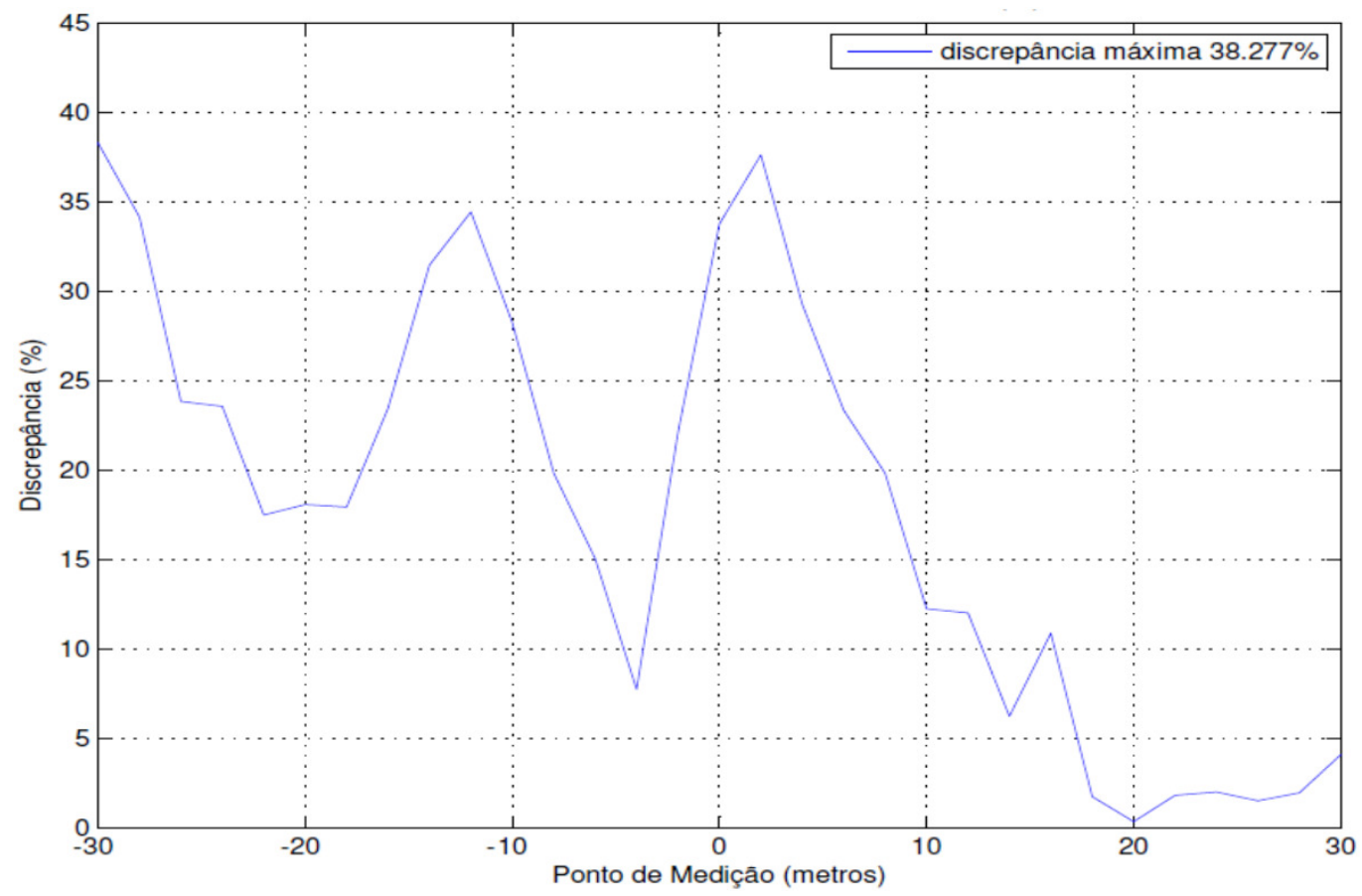

Figura 3 - Discrepância entre valores Calculados e Medidos

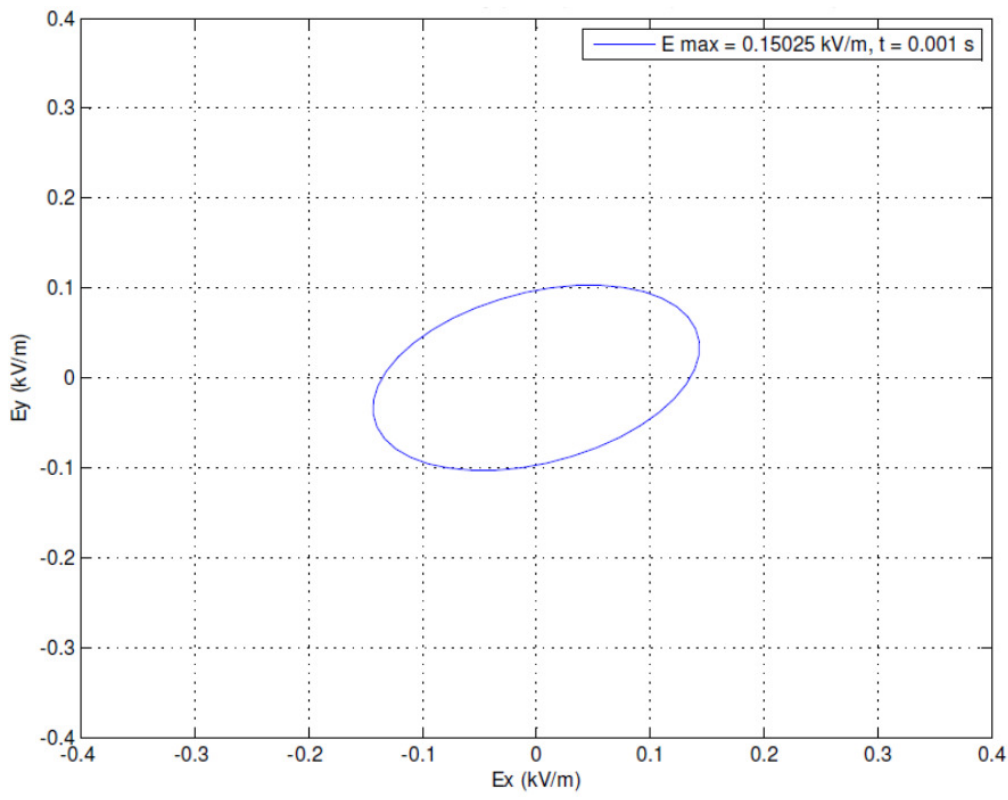

Figura 4 - Trajetória do vetor campo elétrico - $x \mathrm{~N}=0(\mathrm{yN}=1,8 \mathrm{~m})$ 


\section{ARTigo TéCNICO}

Simulou-se a distância mínima do cabo - solo, igual a 12 m, que originava um perfil do campo elétrico com um valor eficaz máximo inferior a $5 \mathrm{kV} / \mathrm{m}$ na zona de segurança, para a altura de $1,8 \mathrm{~m}$.

O perfil do campo magnético e das respectivas componentes no plano (Fig.5) foi calculado para a corrente de $290 \mathrm{~A}$, que se estabeleceu durante a medição do campo magnético desta LAT.
O gráfico da Fig.6 mostra os valores medidos e os valores calculados para o campo magnético, considerando que a corrente atingiu o valor máximo de $290 \mathrm{~A}$.

A carga máxima da LAT Batalha - Pego é de 2500 A, para este perfil o valor calculado para o $B_{\text {ef } \max }$ seria de $7,8 \mu \mathrm{T}$.

Para a distância mínima do cabo - solo de $12 \mathrm{~m}$ e para a carga máxima obtém-se $B_{\text {ef } \max }=49,5 \mu \mathrm{T}$.

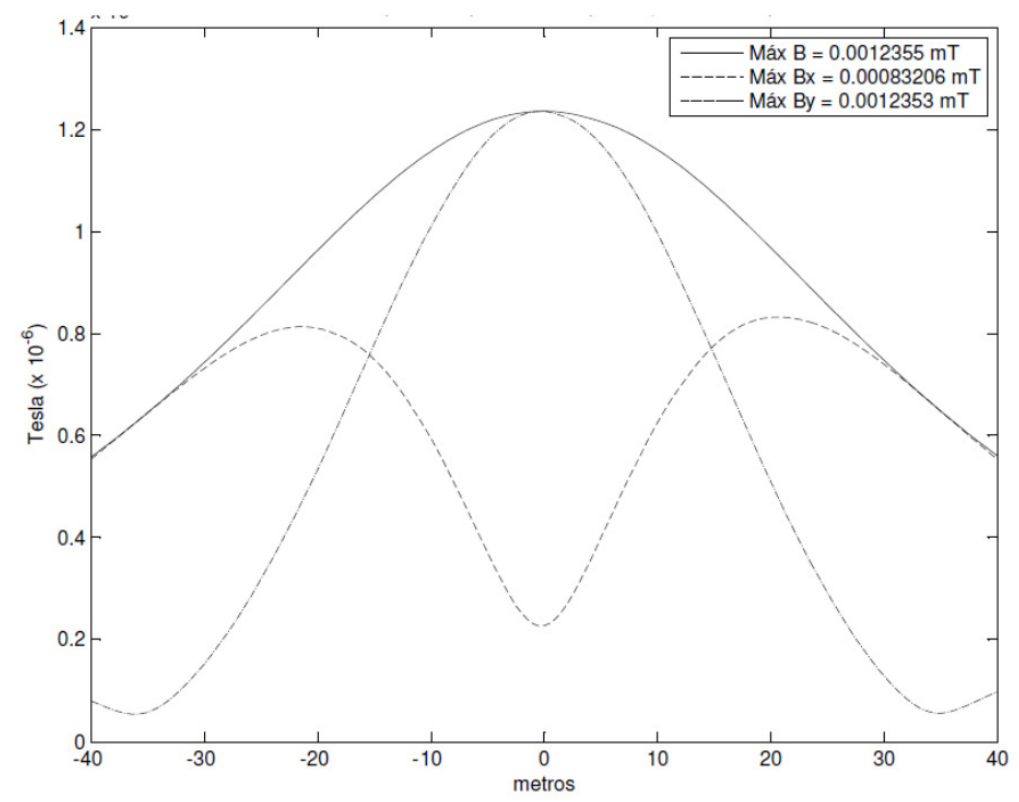

Figura 5 - Perfil do campo magnético máximo e das componentes horizontal e vertical

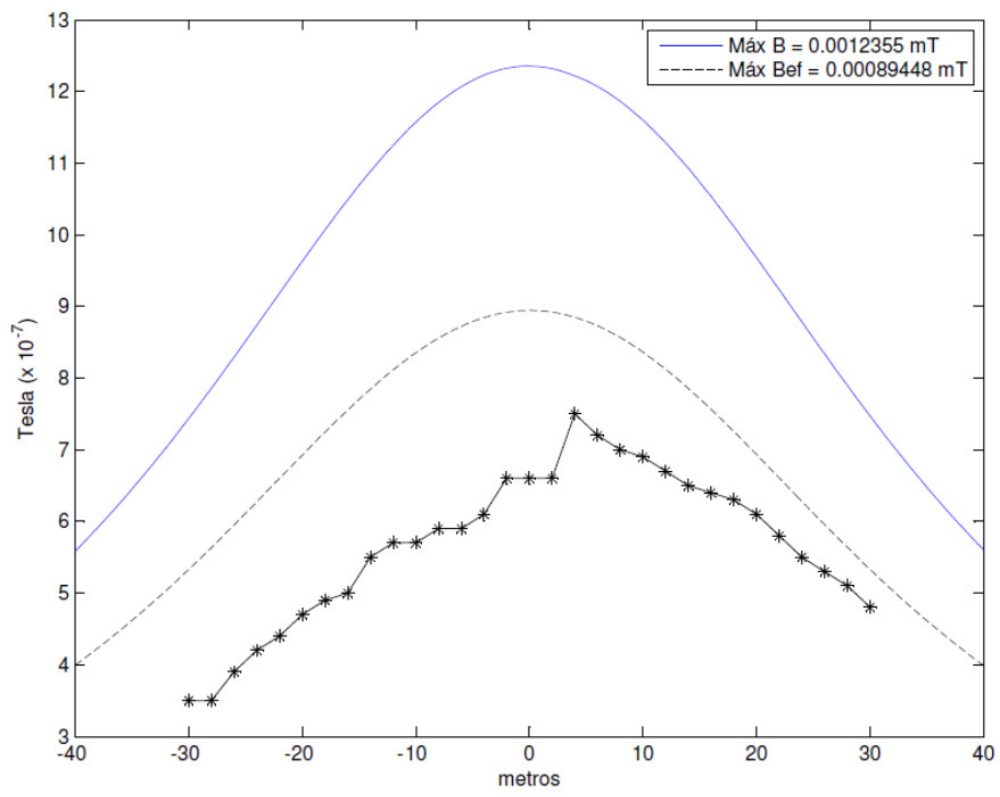

Figura 6 - Perfil do campo magnético para os valores medidos e calculados 
Na Fig.7 apresenta-se a trajetória descrita pelo vetor campo magnético, no ponto $\mathrm{xN}=20 \mathrm{~m}(\mathrm{yN}=1,8 \mathrm{~m})$.

O programa de cálculo foi também aplicado, com sucesso e com resultados excelentes, a outros perfis com medições que apresentavam confiança e fiabilidade, perfil 8 [2] e perfis 17 e 18 do RL 696/04 - AT [3].

\section{Linha Aérea de Transmissão Dupla RM/PR - BATALHA}

Um interessante caso de estudo, que confirmou as potencialidades do cálculo no tempo dos campos.

A LAT dupla RM/PR - Batalha [4] de 220 kV é suportada por postes tipo DL de $8 \mathrm{~m}$ de largura. É uma LAT com dois ternos de configuração vertical, as fases mais próximas do solo ficam à altura aproximada de $17 \mathrm{~m}$. A LAT

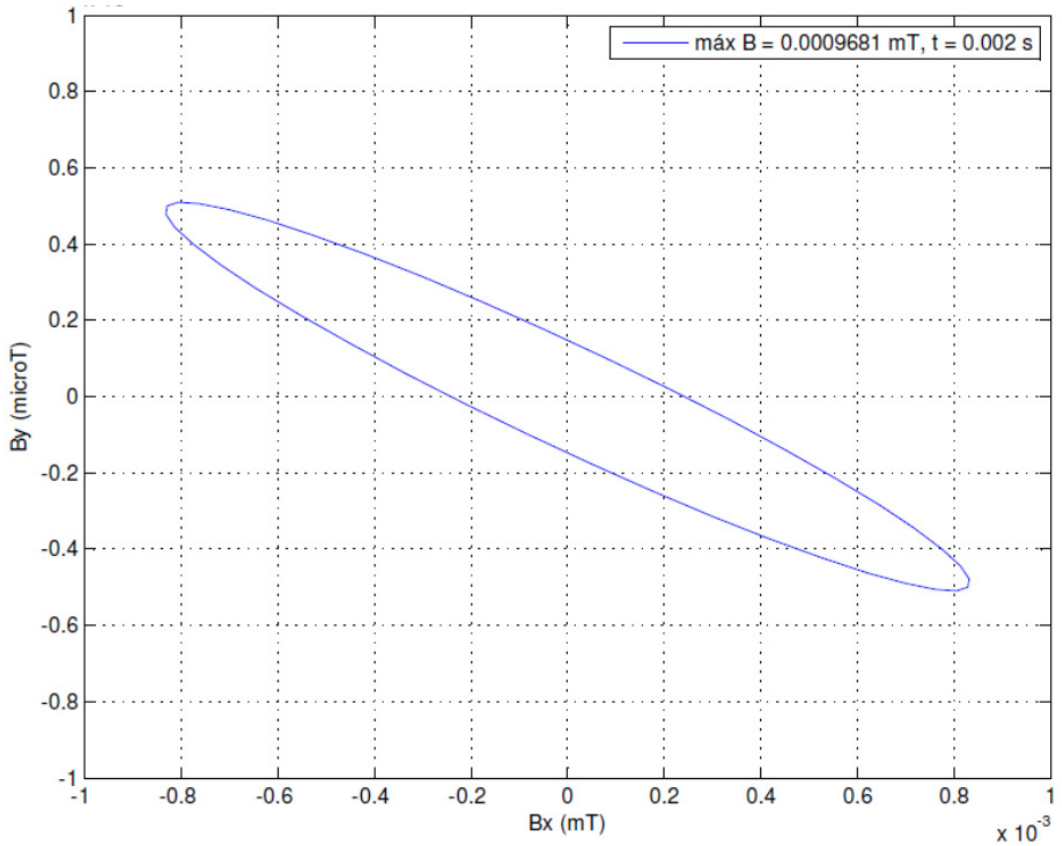
dupla é um troço comum de duas linhas de origens diferentes, LAT Rio Maior Batalha2 e LAT Pereiros/Recarei - Batalha2. As correntes máximas são, respetivamente, $1000 \mathrm{~A}$ e $622 \mathrm{~A}$.

Foi simulada a colocação de baixo para cima das fases a, b, c - c', b', a' das linhas, e considerou-se as duas linhas em fase. A Fig.8 mostra os cálculos obtidos e as medições respectivas efetuadas para o perfil 3 do campo elétrico do relatório 0969/06 - AT [4], sendo o resultado inconsistente.

Figura 7 - Trajetória de campo magnético no ponto $20 \mathrm{~m}$, altura 1,8 m

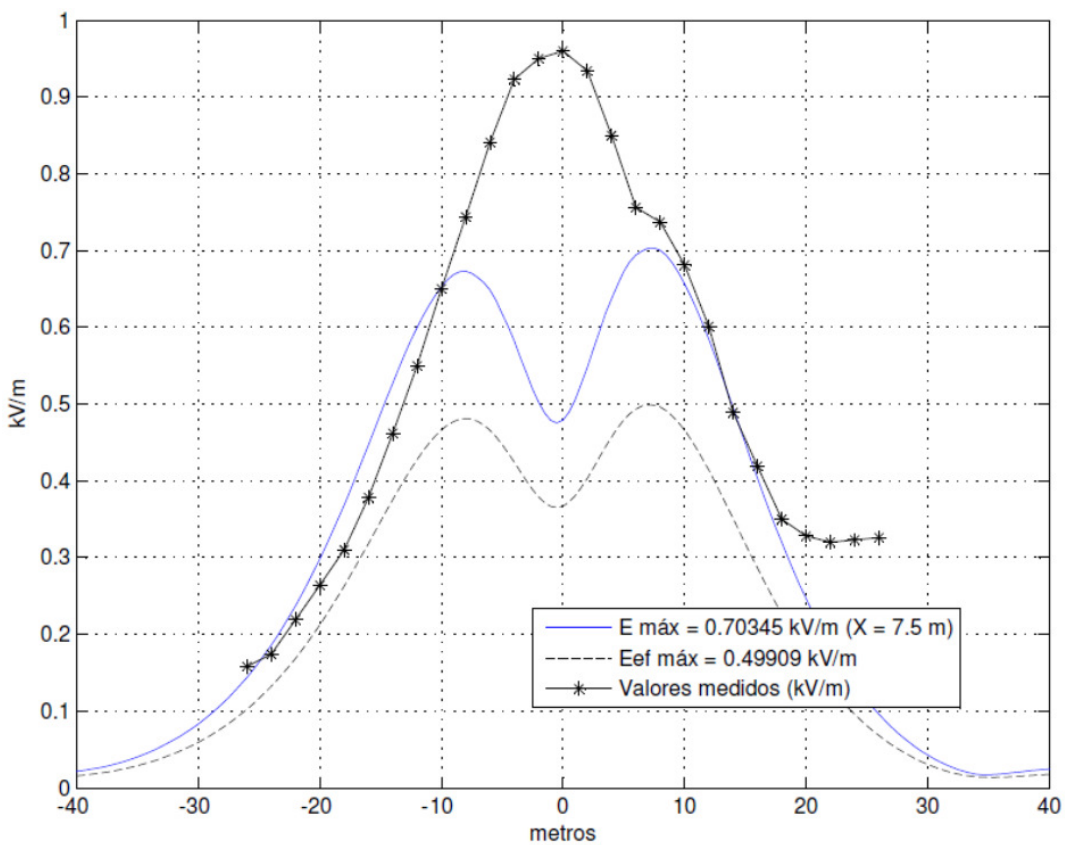

Figura 8 - Perfil do campo elétrico para os valores medidos e calculados - LAT RM/PR - Batalha2 em fase 
É evidente que as linhas aéreas de transmissão, de proveniências diferentes, não estavam em fase quando foram efetuadas as medições.

Tendo sido simulado um desfasamento de cerca de 60 을 entre elas, os resultados obtidos para os perfis dos campos, à altura de 1,8 m, justificam esta situação (Fig.9 e Fig.11).
$\mathrm{Na}$ análise dos valores calculados e medidos do perfil do campo elétrico verificam-se as discrepâncias apresentadas na Fig.10, para além dos $20 \mathrm{~m}$ da linha (Fig.9) o ligeiro aumento dos campos medidos é devido, provavelmente, à existência de duas linhas de $400 \mathrm{kV}$ a cerca de $70 \mathrm{~m}$ para o lado esquerdo e de uma linha de $220 \mathrm{kV}$ a cerca de $50 \mathrm{~m}$ do lado direito [4].

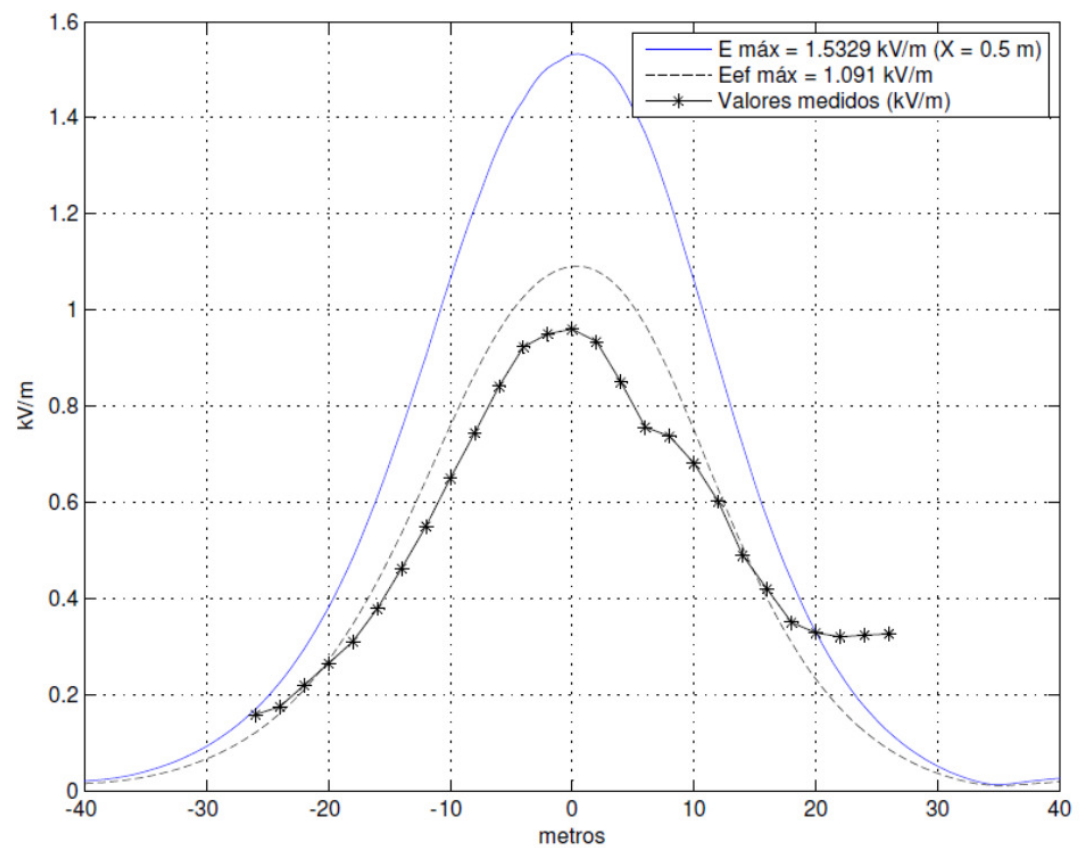

Figura 9 - Perfil do campo elétrico para os valores medidos e calculados - LAT RM/PR - Batalha2 desfasadas

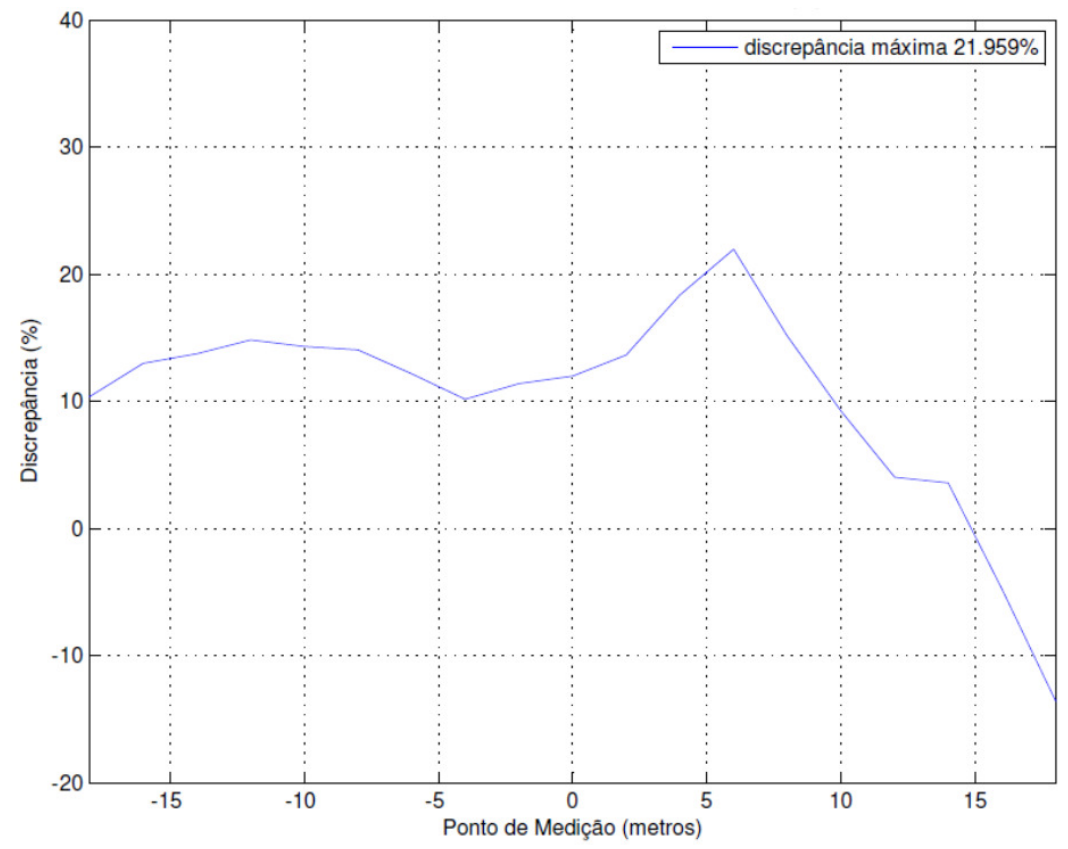

Figura 10 - Discrepância entre valores Calculados e Medidos - linhas RM/PR - Batalha2 desfasadas 
O perfil do campo magnético (Fig.11) foi calculado para as correntes de $140 \mathrm{~A}$ e $55 \mathrm{~A}$ que se estabeleceram durante a medição do campo magnético desta LAT dupla.

Simulou-se a distância mínima do cabo - solo, na condição extrema das LAT estarem desfasadas de 120 ㅇ e os condutores mais próximos do solo estarem em fase, que originava um perfil do campo elétrico com um valor eficaz máximo inferior a $5 \mathrm{kV} / \mathrm{m}$ na zona de segurança e para a altura de 1,8 m. A distância mínima cabo - solo obtida foi de 7 metros.

Para esta distância mínima e para as correntes máximas previstas para as linhas, 1000 A e 622 A, obteve-se o valor eficaz máximo de 25,5 $\mu$ T para o campo magnético.

\section{Linhas Aéreas de 1100 kV}

O programa foi aplicado para o cálculo dos campos de duas Linhas de Ultra Alta Tensão.
Procurou-se obter a distância mínima do cabo - solo que originava um perfil do campo elétrico com um valor eficaz máximo inferior a $5 \mathrm{kV} / \mathrm{m}$ na zona de segurança, ao nível do solo.

Os cálculos dos campos são efetuados para a altura de $1 \mathrm{~m}$.

\section{A. Linha Aérea de Transmissão Kita - Iwaki}

A linha aérea de transmissão Kita - Iwaki [6] de 1100 kV é suportada por postes de $108 \mathrm{~m}$ de altura e $38 \mathrm{~m}$ de largura.

É uma linha dupla com dois ternos de configuração vertical sendo as fases constituídas por feixes de oito condutores, as fases mais próximas do solo ficam à altura de $50 \mathrm{~m}$.

A corrente pode atingir $8000 \mathrm{~A}$, durante períodos de tempo relativamente curtos.

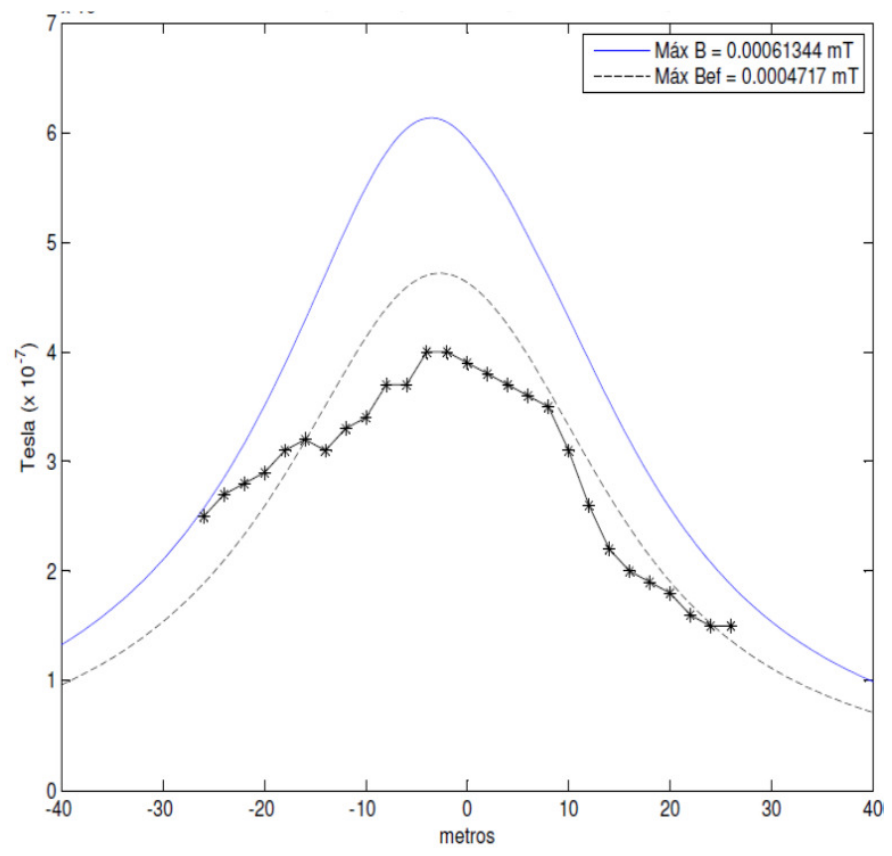

Figura 11 - Perfil do campo magnético para os valores medidos e calculados - LAT RM/PR - Batalha2 desfasadas 
A distância mínima cabo - solo é de $31 \mathrm{~m}$ para que o valor eficaz do campo elétrico não ultrapasse os 5 kV/m (Fig.12).
O perfil do campo magnético foi calculado para correntes de 8000 A e para a mesma distância cabo - solo (Fig.13).

O valor eficaz da componente vertical do campo é

praticamente igual ao valor eficaz do vetor campo.

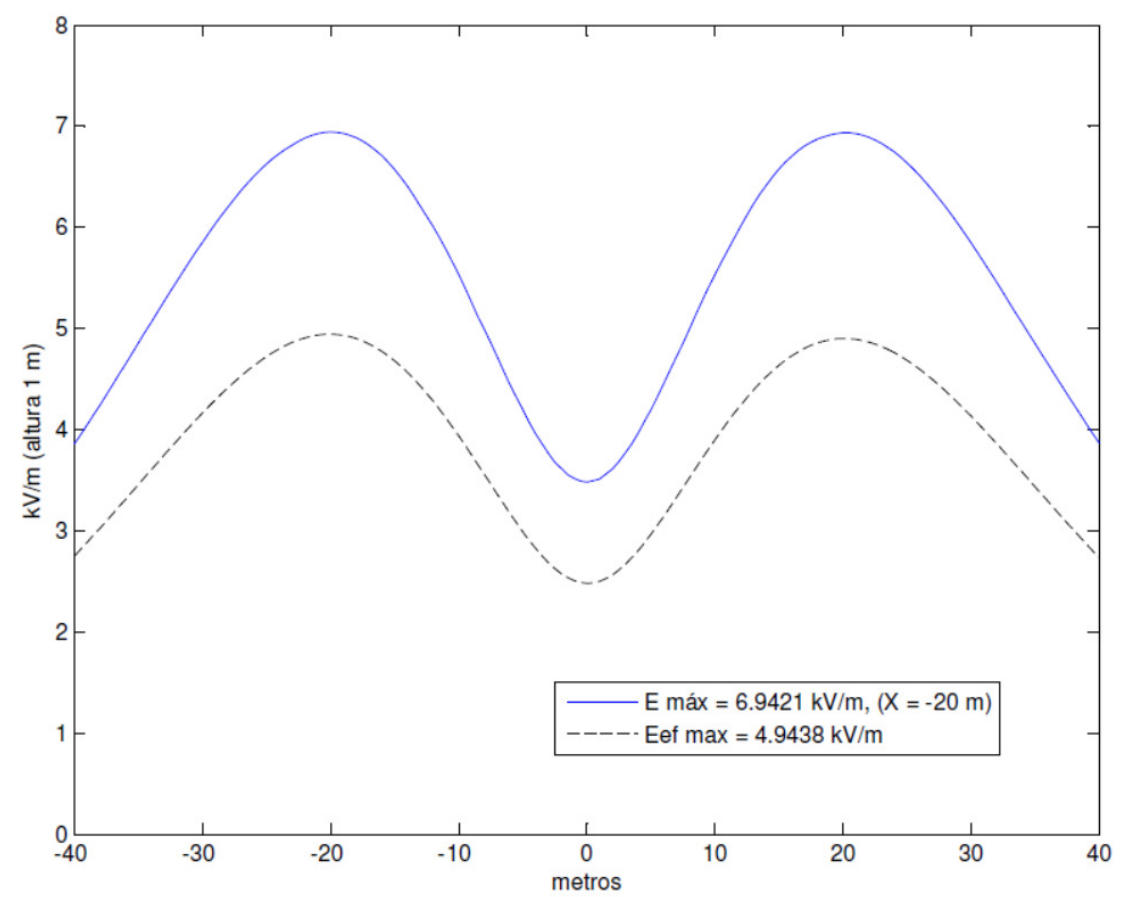

Figura 12 - Perfil do campo elétrico - Distância do cabo - solo de 31 m

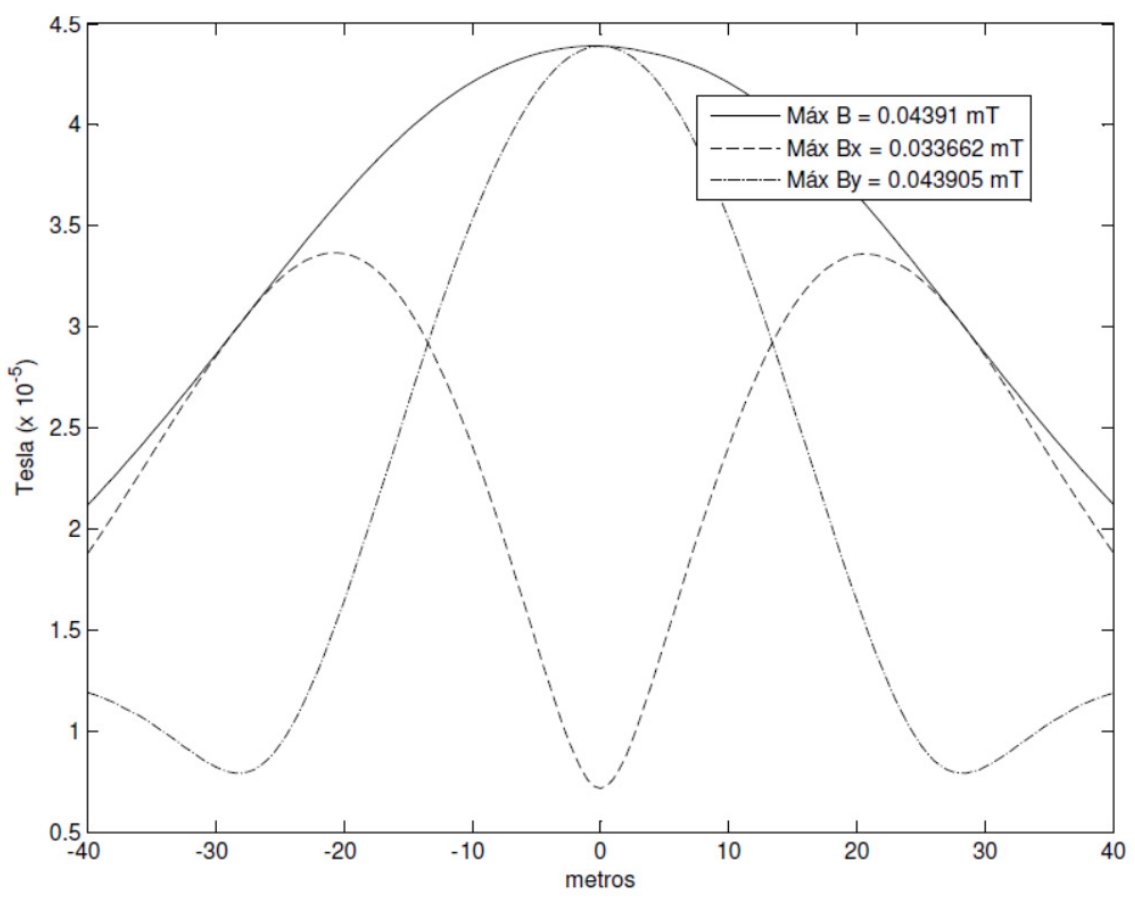

Figura 13 - Perfil do campo magnético máximo e das componentes horizontal e vertical - Distância do cabo - solo de $31 \mathrm{~m}$ 


\section{ARTIGO TÉCNICO}

Foi simulada a situação de um dos ternos verticais se encontrar desligado. Como o equilíbrio da simetria das fases desaparece, o campo elétrico intensifica-se (Fig.14). Situação idêntica à que ocorre quando se aumenta a distância entre os condutores (fases).
A distância mínima do cabo - solo, nestas condições, teria de aumentar para $35 \mathrm{~m}$, para que na zona de segurança o valor eficaz do campo elétrico não ultrapassasse o valor de referência. O cálculo do perfil do campo magnético, naquela situação, está representado na Fig.15.

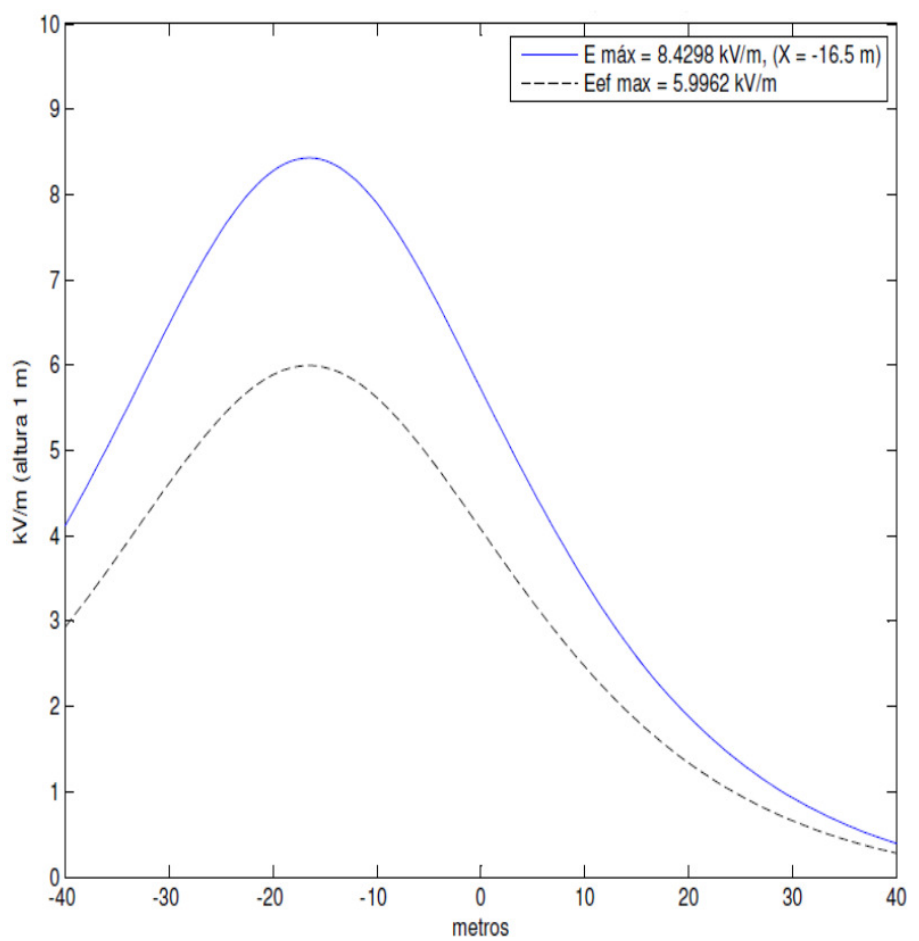

Figura 14 - Perfil do campo elétrico (1 terno vertical)

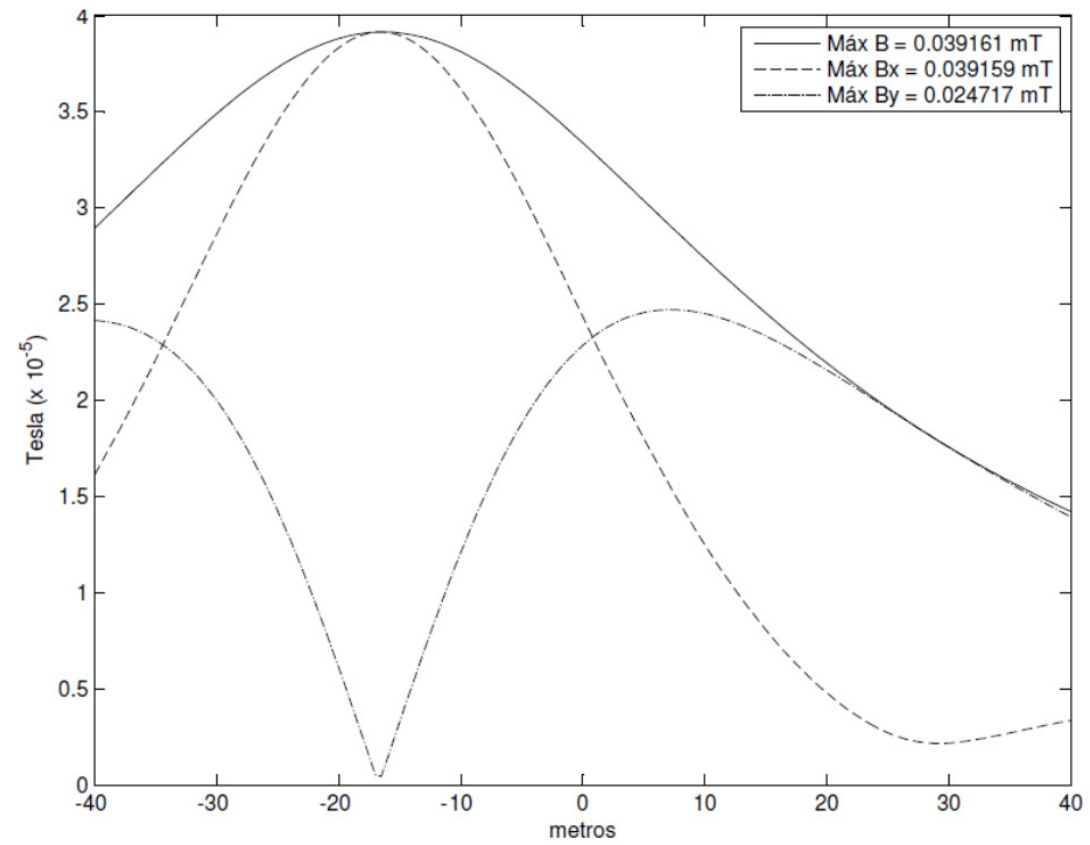

Figura 15 - Perfil do campo magnético máximo (1 terno vertical) e das componentes horizontal e vertical 


\section{B. Linha Aérea de Transmissão Ekibastuz - Kokchetav}

A LAT Ekibastruz - Kokchetav [6] de 1100 kV é suportada por postes de $44 \mathrm{~m}$ de altura e $48 \mathrm{~m}$ de largura. É constituída por um terno de configuração horizontal sendo as fases constituídas por feixes de oito condutores, as fases exteriores, que se encontram mais próximas do solo, ficam à altura de $32 \mathrm{~m}$. A corrente pode atingir $8000 \mathrm{~A}$.

Não existe distância mínima cabo - solo para que o valor eficaz do campo elétrico não ultrapasse os $5 \mathrm{kV} / \mathrm{m}$.
Para a distância teórica de 32 metros o valor eficaz máximo calculado é igual a 5,4 kV/m (Fig.16).

Para que o valor eficaz do campo fosse inferior a $5 \mathrm{kV} / \mathrm{m}$, na zona de segurança, a distância mínima do cabo - solo teria de ser de $34 \mathrm{~m}$.

O perfil do campo magnético calculado para os pontos com 1 $\mathrm{m}$ de altura, supondo que os condutores se encontram à distância de $32 \mathrm{~m}$ do solo e a corrente é de $8000 \mathrm{~A}$, está representado na Fig.17.

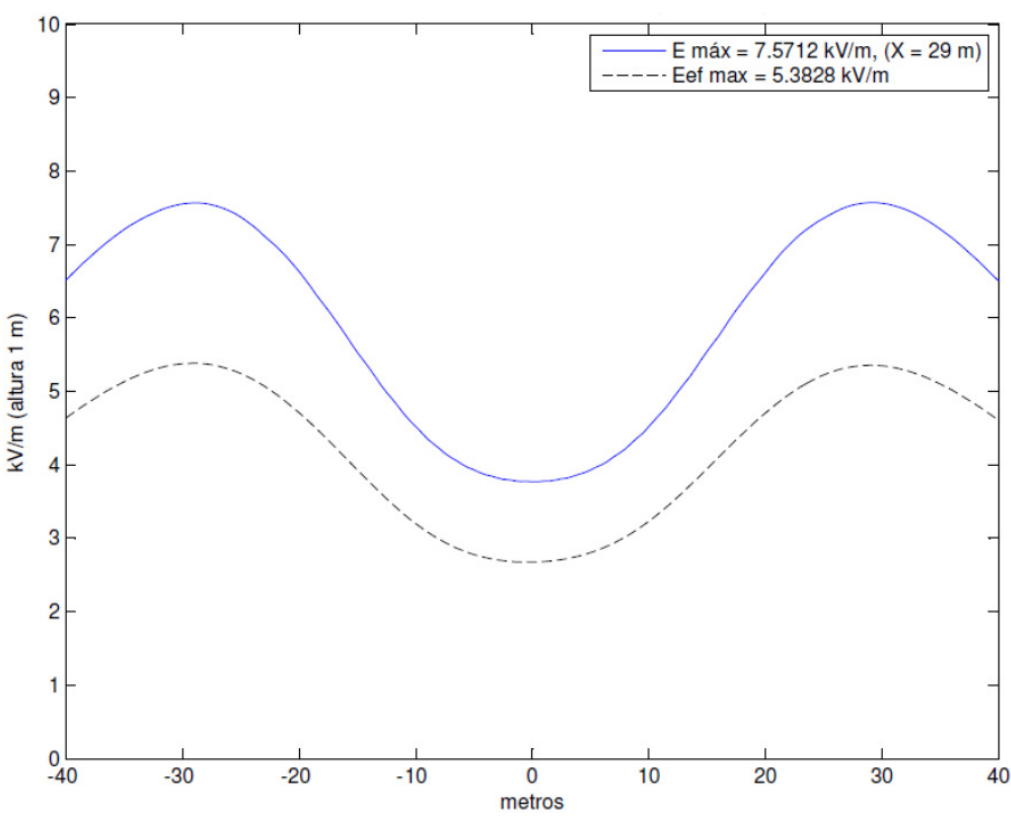

Figura 16 - Perfil do campo elétrico - Distância do cabo - solo de 32 m

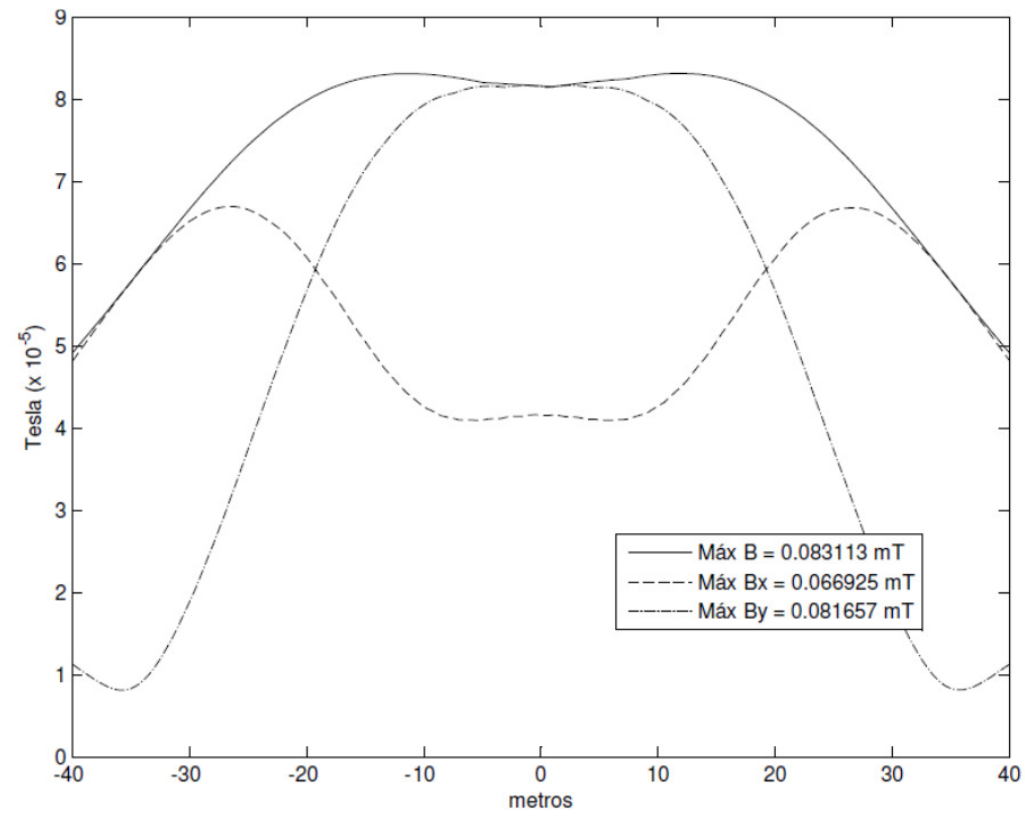

Figura 17 - Perfil do campo magnético máximo e das componentes horizontal e vertical - Distância do cabo - solo de 32 m 
Em termos gerais, pode-se concluir que o campo elétrico máximo no solo é menor para a configuração triangular equilateral das LAT e maior para as configurações vertical e horizontal. O campo elétrico fora da faixa de segurança é mais pequeno para a configuração vertical e é maior para a configuração horizontal.

É inconsequente tentar parametrizar o campo elétrico em função das dimensões geométricas das LAT, mesmo que não se considere a condutividade do terreno, basta a diversidade da tipologia dos apoios e o número de condutores que constituem as fases, para inviabilizar essa intenção. É apenas possível prever uma distância mínima que cumpra as condições de segurança estudadas.

O efeito da dimensão do condutor pode ser avaliado, no caso de feixes nas fases, usando o raio equivalente [5].

O condutor equivalente tem a mesma carga elétrica total por unidade de comprimento da fase, o que causa o mesmo campo no solo.

Verifica-se que a duplicação teórica do raio equivalente provoca um aumento de cerca de $15 \%$ no valor do campo eléctrico.

Os cabos de guarda (CG) praticamente não influenciam o campo elétrico ao nível do solo.

A não existência teórica dos CG aumentava o campo elétrico no solo no máximo de $4 \%$, para a configuração horizontal de $1100 \mathrm{kV}$.

\section{Conclusão}

A utilização de valores eficazes nos potenciais e nas cargas, na simulação dos campos elétricos, utilizando o método da simulação das cargas e apresentado na maioria das publicações sobre o assunto, mostra-se adequado. Apesar da utilização de campos estacionários originar a obtenção de valores eficazes aproximados para os campos elétricos.
A utilização de valores instantâneos no método da simulação das cargas apresenta resultados teóricos, em condições ideais, próximos ou coincidentes com os valores reais e medidos.

O método utilizado para o cálculo mostrou-se eficaz e preciso na determinação do perfil do campo elétrico ao nível do solo em LAT, os resultados são obtidos de forma rápida e com relativa simplicidade, os valores obtidos estão otimizados devido às condições ideais consideradas, solo plano, horizontal, infinito e condutor perfeito e condutores teoricamente paralelos entre si e ao solo (o efeito de flecha desprezado quando considerado o ponto mais baixo da catenária), o que poderá ser corrigido com um conhecimento mais detalhado das condições de implementação da LAT.

O campo magnético em pontos igualmente afastados de um sistema trifásico simétrico de correntes é nulo devido à sua natureza rotacional.

Em pontos suficientemente afastados do sistema trifásico, devido à assimetria da colocação das fases, o valor do campo é residual.

A utilização dos valores eficazes das correntes na simulação computacional do campo magnético não é a mais adequada, obtendo valores excessivamente divergentes.

A utilização de valores instantâneos permite obter valores coincidentes com os valores teóricos previstos.

Os campos elétricos e magnéticos máximos, recomendados para Público Permanente, são respetivamente; $5 \mathrm{kV} / \mathrm{m}$ (RMS) e 0,100 mT (RMS) [6].

De acordo com estes valores, a grande maioria das LAT, com tensões nominais inferiores a 800 kV, não necessitam de zona de segurança para o campo elétrico, desde que cumpram uma distância mínima ao solo (público). 
A distância mínima ao solo para uma linha típica de 800 kV, com configuração horizontal, considerando uma distância entre fases de $16 \mathrm{~m}$ e as fases constituídas por 4 condutores, é de $23 \mathrm{~m}$.

Os campos magnéticos das linhas aéreas de transmissão têm valores reduzidos e inferiores ao limite considerado como perigoso para a saúde pública.

O campo magnético das linhas aéreas de transmissão não tem influência na segurança do público permanente, de acordo com as recomendações.
Estudos futuros poderão reavaliar os procedimentos de medição e de cálculo com a finalidade de minimizar as discrepâncias encontradas, e a implementação de novos conceitos ao programa tornando-o mais abrangente neste tipo de cálculo.

\section{Referências}

[1] H. Singer, H. Steinbigler, e P. Weiss, "A charge simulation method for the calculation of high voltage fields," IEEE Trans., vol. PAS-93, pp. 1660-1668, January 1974.

[2] REN - Portugal, Informação Técnica, Monitorização - Campos Eléctrico e Magnético, Relatório da Linha Batalha - Pego, Novembro 2007.

[3] REN - Portugal, Informação Técnica, Monitorização - Campos Eléctrico e Magnético, Relatório da Linha Fanhões - Alto Mira IV/V a 400 kV, Novembro 2004.

[4] REN - Portugal, Informação Técnica, Monitorização - Campos Eléctrico e Magnético, Relatório do Troço comum às Linhas Batalha - Recarei e Batalha - Rio Maior 3, Novembro 2006.

[5] J. S. Paiva, "Redes de Energia Eléctrica - uma análise sistémica”, ISTPress, Lisboa, Abril 2005.

[6] Siemens Energy Sector, UHV AC Entwicklung uber 1100 kV, L.-R. Janicke, at all, Hannover Messe, April 2008.

[7] Council Recommendation on the Limitation of Exposure of the General Public to Electromagnetic Fields $0 \mathrm{~Hz}-300 \mathrm{GHz}$, 2188a Reunião do Conselho da União Europeia, Junho 1999.

\section{Agradecimentos}

Agradeço a REN - Rede Eléctrica Nacional toda a disponibilidade no acesso às publicações da Informação Técnica, e na colaboração do Centro de Informação Técnica da REN para a utilização dos dados técnicos e medições.

http://www.centrodeinformacao.ren.pt/PT/InformacaoTecnica/Paginas/MonitorizacaoCamposElectricoMagnetico.aspx

Obs:

Artigo publicado na Revista: LATIN AMERICA TRANSACTIONS, VOL. 10, NO. 4, JUNE 2012 